\title{
New approaches in the analysis of Dark Matter direct detection data: scratching below the surface of the most general WIMP parameter space.
}

\author{
Scopel, Stefano*; Yoon, Kook-Hyun; Yoon, Jong-Hyun \\ Physics Department, Sogang University, \\ Seoul, 121-742, South Korea \\ *E-mail: scopel@sogang.ac.kr
}

\begin{abstract}
We show that compatibility between the DAMA modulation result (as well as less statistically significant excesses such as the CDMS Silicon effect and the excess claimed by CRESST) with constraints from other experiments can be achieved by extending the analysis of direct detection data beyond the standard elastic scattering of a WIMP off nuclei with a spin-dependent or a spin-independent cross section and with a velocity distribution as predicted by the Isothermal Sphere model. To do so we discuss several new approaches for the analysis of Dark Matter direct detection data, with the goal to remove or reduce its dependence on specific theoretical assumptions, and to extend its scope: the factorization approach of astrophysics uncertainties, the classification and study of WIMP-nucleon interactions within non-relativistic field theory, inelastic scattering and isovector-coupling cancellations including subdominant two-nucleon NLO effects. Typically, combining two or more of these ingredients can lead to conclusions which are very different to what usually claimed in the literature. This shows that we are only starting now to scratch the surface of the most general WIMP direct detection parameter space.

Keywords: Dark Matter; direct detection
\end{abstract}

\section{Content}

Many underground experiments are currently searching for Weakly Interacting Massive Particles (WIMPs), which are the most popular candidates to provide the Dark Matter (DM) which is believed to make up $27 \%$ of the total mass density of the Universe, and more than $90 \%$ of the halo of our Galaxy. One of them (DAMA $\frac{1}{*}$ ) has been observing for more than 15 years a yearly modulation effect in the low part of its energy spectrum which is consistent with that expected due to the Earth rotation around the Sun from the elastic scattering of WIMPs off the sodium iodide nuclei that constitute the crystals of its scintillators. Many experimental collaborations using nuclear targets different from $\mathrm{NaI}$ and various background-subtraction techniques to look for WIMP-elastic scattering (including LUX $\underline{2}$, SuperCDMS $\underline{3}$, COUPP $\stackrel{4}{\underline{4}}$, PICASSO $\stackrel{5}{=}$ ) have failed to observe any anomaly so far, implying severe constraints on the most popular WIMP scenarios used to explain the DAMA excess. A similar situation arises when confronting the excesses claimed by CDMS-Si $\underline{\underline{6}}$ ad CRESST $^{\underline{7}}$ with the same constraints.

However, besides the fact that several experimental uncertainties might still be advocated to question the robustness of these bounds, in most cases such conclusions are drawn by analysing direct detection data assuming elastic scattering of a WIMP off nuclei with a spin-dependent or a spin-independent cross section and with a WIMP velocity distribution $f(\vec{v})$ as predicted by the Isothermal Sphere model. 
These assumtions may be well motivated, but represent only a very small part of the possible and experimentally viable options.

For instance, if the DAMA effect can be explained by scatterings of WIMPS off sodium such constraints can be potentially relaxed by considering models where the nuclear response function of sodium on the WIMP interaction is enhanced compared to that of the nuclei used to obtain the experimental bounds (germanium, xenon, fluorine).

In light of the situation summarized above several new directions have been explored in the recent past both to remove as much as possible the dependence on specific theoretical assumptions (both of particle-physics and astrophysical origin) from the analysis of DM direct detection data and to extend its scope to a wider class of models.

Starting from ${ }^{8}$ a general strategy has been developed to factor out the dependence on $f(\vec{v})$ of the expected WIMP-nucleus differential rate $d R / d E_{R}$ at the given recoil energy $E_{R}$. This approach exploits the fact that $d R / d E_{R}$ depends on $f(\vec{v})$ only through the minimal velocity $v_{\min }$ that the WIMP must have to deposit at least $E_{R}$, i.e.:

$$
\frac{d R}{d E_{R}} \propto \eta\left(v_{\min }\right) \equiv \int_{|\vec{v}|>v_{\min }} \frac{f(\vec{v})}{|\vec{v}|} d^{3} v .
$$

By mapping recoil energies $E_{R}$ into same ranges of $v_{\min }$ the dependence on $\eta\left(v_{\min }\right)$ and so on $f(\vec{v})$ cancels out in the ratio of expected rates on different targets. Since the mapping between $E_{R}$ and $v_{\text {min }}$ depends on the nuclear mass the factorization of $\eta\left(v_{\text {min }}\right)$ is only possible in the case of detectors with a single nuclear target, or for which the expected rate is dominated by scatterings on a single target. In 1.14 , after discussing the applicability and limitations of this method to the DAMA data, we have extended this procedure to the case when a constraining experiment contains different targets.

In particular, a scenario proposed to alleviate the tension among different direct detection experiments is Inelastic Dark Matter (IDM). In this class of models a Dark Matter (DM) particle $\chi$ of mass $m_{D M}$ interacts with atomic nuclei exclusively by up-scattering to a second state $\chi^{\prime}$ with mass $m_{D M}^{\prime}=m_{D M}+\delta$. In the case of exothermic Dark Matter $\delta<0$ is also possible: in this case the particle $\chi$ is metastable and down-scatters to a lighter state $\chi^{\prime}$. In the IDM scenario the halomodel factorization approach is more complicated that in the elastic case, because in presence of a mass splitting $\delta \neq 0$ the mapping between the nuclear recoil energy $E_{R}$ and the minimal velocity $v_{\min }$ that the incoming WIMP needs to have to deposit $E_{R}$ becomes more involved than in the elastic case. $\operatorname{In}^{\underline{9}}$ we provided the first systematic analysis of IDM for a spin-independent cross section, i.e. scaling as:

$$
\sigma \propto\left[Z f_{p}+(A-Z) f_{n}\right]^{2},
$$

with $f_{p, n}$ the WIMP couplings to protons and neutrons, respectively and $A, Z$ the nuclear mass and atomic number. Including all available data and making use of the 
factorization property of the halo-model dependence we introduced some strategies to determine regions in the IDM parameter space where the tension existing among different experimental results can be eliminated or at least alleviated: indeed, as shown in Fig 1, compatibility between DAMA or CDMS-Si and all other constraints can be achieved. In the same analysis, we also showed that the same thing can be also obtained for the excess measured by $\operatorname{CRESST}^{\underline{7}}$ (we note there that this result, which holds for $m_{\chi} \gtrsim 40 \mathrm{GeV}$, is still true after the low-background re-analysis of Ref. 10 ).

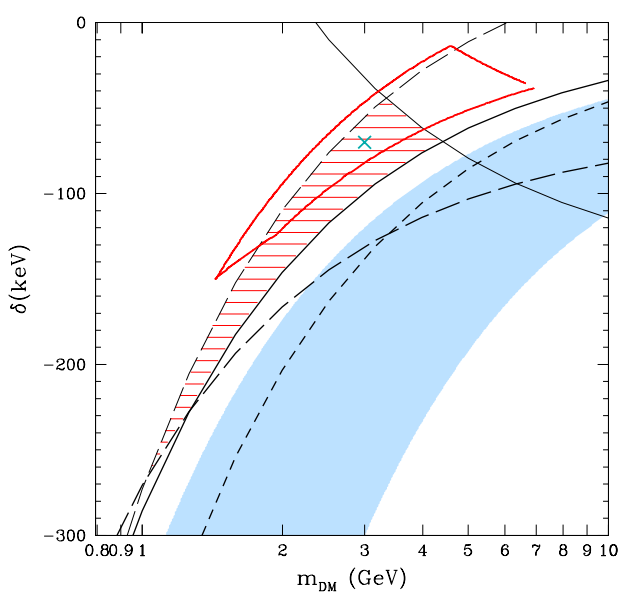

Fig. 1. Mass splitting $\delta=m_{D M}^{\prime}-m_{D M}$ as a function of $m_{D M}$. The closed contour bounded by the solid (red) line represents the IDM parameter space where the modulation effect measured by DAMA is compatible to other constraints, while the horizontally (red) hatched area represents

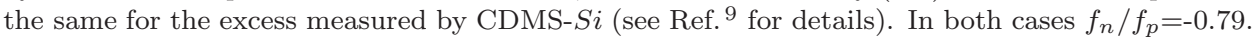

On the other hand, in $\frac{13}{2}$ we have considered the effect of the inclusion of the NLO corrections calculated in $\frac{12}{2}$ in a specific scenario of light IDM with the same scaling of the cross section as in Eq. (2) for which $f_{n}, f_{p}$ violate isospin symmetry (Isospinviolating Dark Matter, IVDM) leading to a suppression of the WIMP cross section off Germanium targets. By incorporating this scenario in the halo-independent approach also in this case, as shown in Fig 2, a region of the parameter space can be achieved where the CDMS-Si excess is compatible to other constraint. This is obtained for $m_{\chi} \lesssim 4 \mathrm{GeV}$ and $\delta<0$ (exothermic DM).

One of the most popular scenarios for WIMP-nucleus scattering is a spindependent interaction where the WIMP particle $\chi$ is a fermion (either Dirac or Majorana) that recoils on the target nucleus $T$ through it coupling to the spin $\vec{S}_{N}$ of nucleons $N=(p, n)$ :

$$
\mathcal{L}_{i n t} \propto \vec{S}_{\chi} \cdot \vec{S}_{N}=c^{p} \vec{S}_{\chi} \cdot \vec{S}_{p}+c^{n} \vec{S}_{\chi} \cdot \vec{S}_{n}
$$

Among the main motivations of such scenario is the fact that the most stringent 


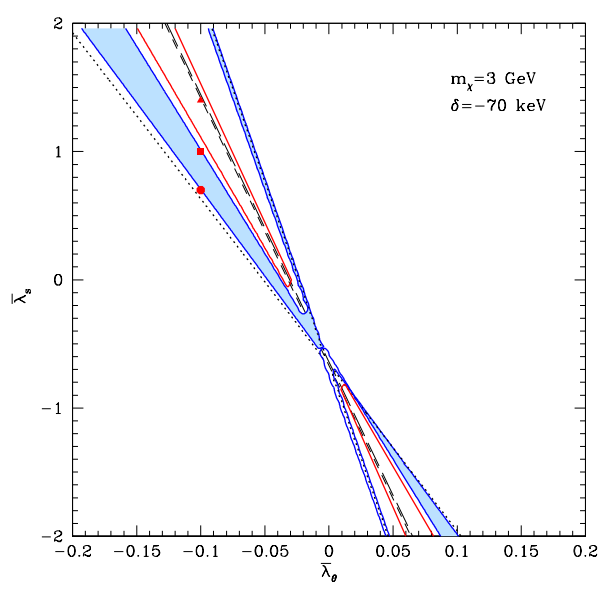

Fig. 2. In this plot for any choice of $\bar{\lambda}_{\theta}$ and $\bar{\lambda}_{s}$, which parametrize the metastable WIMP couplings to heavy quarks and the strange quark, respectively, the ratio of the corresponding couplings to light quarks $\bar{\lambda}_{d} / \bar{\lambda}_{u}$ is fixed to maximize the compatibility between CDMS-Si and SuperCDMS. The shaded regions represent the parameter space where CDMS-Si and SuperCDMS are mutually compatible including NLO corrections to the cross section while at the same time the metastable state $\chi$ can be a thermal relic (i.e. $\Omega_{\chi} h^{2} \leq 0.12$ ). The dotted curve represents the condition $\Omega_{\chi} h^{2}=0.12$ calculated using the LO instead of the NLO scaling law for the expected rate. The inner solid (red) line corresponds to $\tau=1 / \Gamma=4 \times 10^{26}$ seconds, the lifetime of the metastable state $\chi$. See Ref. 13 for details.

bounds on the interpretation of the DAMA effect in terms of WIMP-nuclei scatterings arise today from detectors using xenon (LUX $\underline{2}$ ) and germanium (SuperCDMS $\underline{3}$ ) whose spin is mostly originated by an unpaired neutron, while both sodium and iodine in DAMA have an unpaired proton: if the WIMP effective coupling to neutrons $c^{n}$ is suppressed compared to that on protons $c^{p}$ this class of bounds can be evaded. However this scenario is presently constrained by droplet detectors (including COUPP $\underline{4}$ ) and bubble chambers (including PICASSO $\underline{\underline{5}}$ ) which all use nuclear targets with an unpaired proton (in particular, they all contain ${ }^{19} F$ ). As a consequence, this class of experiments have been shown to rule out the scenario of Eq. (3) also for $c^{n} \ll c^{p}$ when standard assumptions are made on the WIMP local density and velocity distribution in our Galaxy $\underline{15}$.

In light of this, in Ref. $\underline{14}$ we extended the analysis of spin-dependent WIMPnucleus interactions. The most general WIMP-nucleus spin-dependent interactions ca be singled out by making use of the non-relativistic Effective Field Theory (EFT) approach of Ref. $\underline{\underline{11}}$. According to $\underline{\underline{11}}$ the most general Hamiltonian density for the WIMP-nucleon process can be expressed in terms of a combination of five Hermitian operators which act on the two-particle Hilbert space spanned by tensor products of WIMP and nucleon states; including terms that are at most linear in the nuclear and WIMP spins and quadratic on the WIMP incoming velocity, the most general Hamiltonian density describing the WIMP-nucleus interaction can be written in 


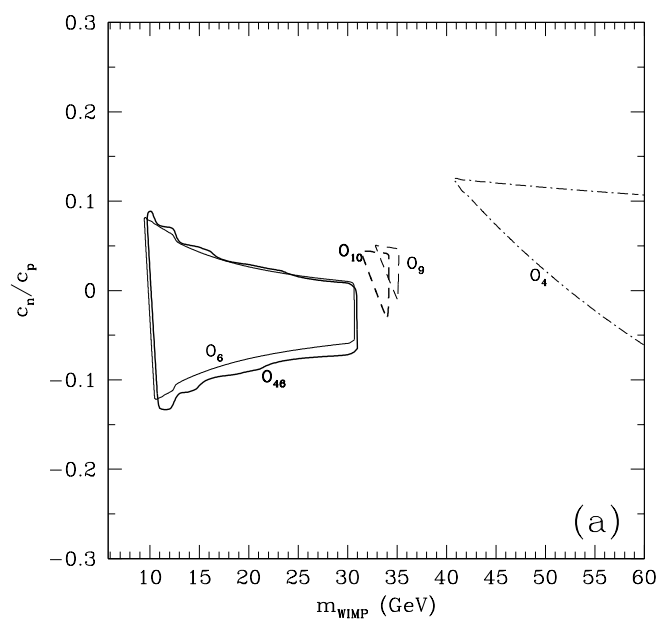

Fig. 3. Contour plot in the $m_{W I M P}-c_{i}^{n} / c_{i}^{p}$ plane for the compatibility factor $\mathcal{D}$ defined in Eqs. $(5.1,5.2)$ of Ref.14. $\mathcal{D} \leq 1$ implies compatibility between DAMA and other constraints. The constant value $\mathcal{D}=1$ is shown for models $\mathcal{O}_{i}, i=6,46,9,10$ (which represent generalized spindependent interactions including explicit momentum and velocity dependence), while a value close to the minimum $(\mathcal{D}=1.7)$ is plotted for $\mathcal{O}_{4}$, which represents the standard spin-dependent interaction, for which DAMA and other constraints cannot be reconciled.

terms of 15 non-relativistic quantum mechanical operators. We used this approach to classify the most general spin-dependent WIMP-nucleus interactions, and within this class of models we discussed the viability of an interpretation of the DAMA modulation result in terms of a WIMP signal, using a halo-independent approach.

Our main conclusions were that, although several relativistic EFT's can lead to a spin-dependent cross section, in some cases with an explicit, non-negligible dependence on the WIMP incoming velocity, three main scenarios can be singled out which approximately encompass them all, and that only differ by their explicit dependence on the transferred momentum. They are represented by models $\mathcal{O}_{6}$ $\left(\simeq \mathcal{O}_{46}\right), \mathcal{O}_{9}\left(\simeq \mathcal{O}_{10}\right)$, and $\mathcal{O}_{4}$ in Fig. 3. For two of them compatibility between DAMA and other constraints can be achieved for a WIMP mass below $30 \mathrm{GeV}$, but only for a WIMP velocity distribution in the halo of our Galaxy which departs from a Maxwellian. This is achieved by combining a suppression of the WIMP effective coupling to neutrons (to evade constraints from xenon and germanium detectors) to an explicit quadratic or quartic dependence of the cross section on the transferred momentum (that leads to a relative enhancement of the expected rate off sodium in DAMA compared to that off fluorine in droplet detectors and bubble chambers). For larger WIMP masses the same scenarios are excluded by scatterings off iodine in COUPP.

The results summarized in this presentation show that, when different new approaches such as the halo-dependence factorization, non-relativistic EFT and inelastic scattering are combined together, conclusions which are very different to 
what usually claimed in the literature can be drawn. This shows that we are only starting now to scratch the surface of the most general WIMP direct detection parameter space.

\section{References}

1. R. Bernabei et al. [DAMA and LIBRA Collaborations], Eur. Phys. J. C 67, 39 (2010) [arXiv:1002.1028 [astro-ph.GA]].

2. D. S. Akerib et al. [LUX Collaboration], Phys. Rev. Lett. 112, no. 9, 091303 (2014) arXiv:1310.8214 [astro-ph.CO]].

3. R. Agnese et al. [SuperCDMS Collaboration], arXiv:1402.7137 [hep-ex].

4. E. Behnke et al. [COUPP Collaboration], Phys. Rev. D 86, no. 5, 052001 (2012) [Erratum-ibid. D 90, no. 7, 079902 (2014)] arXiv:1204.3094 [astro-ph.CO]].

5. S. Archambault et al. [PICASSO Collaboration], Phys. Lett. B 711, 153 (2012) arXiv:1202.1240 [hep-ex]].

6. R. Agnese et al. [CDMS Collaboration], Phys. Rev. Lett. 111, 251301 (2013) arXiv:1304.4279 [hep-ex]].

7. G. Angloher, M. Bauer, I. Bavykina, A. Bento, C. Bucci, C. Ciemniak, G. Deuter and F. von Feilitzsch et al., Eur. Phys. J. C 72, 1971 (2012) arXiv:1109.0702 [astro-ph.CO]].

8. P. J. Fox, J. Liu and N. Weiner, Phys. Rev. D 83, 103514 (2011) arXiv:1011.1915 [hep-ph]].

9. S. Scopel and K. Yoon, JCAP 1408, 060 (2014) arXiv:1405.0364 [astroph.CO]].

10. G. Angloher et al. [CRESST-II Collaboration], Eur. Phys. J. C 74, no. 12, 3184 (2014) doi:10.1140/epjc/s10052-014-3184-9 arXiv:1407.3146 [astro-ph.CO]].

11. A. L. Fitzpatrick, W. Haxton, E. Katz, N. Lubbers and Y. Xu, JCAP 1302, 004 (2013) arXiv:1203.3542 [hep-ph]]; N. Anand, A. L. Fitzpatrick and W. C. Haxton, Phys. Rev. C 89, no. 6, 065501 (2014) arXiv:1308.6288 [hep-ph]].

12. V. Cirigliano, M. L. Graesser and G. Ovanesyan, JHEP 1210, 025 (2012) arXiv:1205.2695 [hep-ph]]; V. Cirigliano, M. L. Graesser, G. Ovanesyan and I. M. Shoemaker, Phys. Lett. B 739, 293 (2014) arXiv:1311.5886 [hep-ph]].

13. S. Scopel and J. H. Yoon, Phys. Rev. D 91, no. 1, 015019 (2015) arXiv:1411.3683 [hep-ph]].

14. S. Scopel, J. H. Yoon and K. Yoon, arXiv:1505.01926 [astro-ph.CO].

15. E. Del Nobile, G. B. Gelmini, A. Georgescu and J. H. Huh, arXiv:1502.07682 [hep-ph]. 